\title{
Removal and Reconstitution of the Carotenoid Antenna of Xanthorhodopsin
}

\author{
Eleonora S. Imasheva $\cdot$ Sergei P. Balashov $\cdot$ \\ Jennifer M. Wang $\cdot$ Janos K. Lanyi
}

Received: 9 September 2010/Accepted: 5 November 2010/Published online: 21 November 2010

(C) The Author(s) 2010. This article is published with open access at Springerlink.com

\begin{abstract}
Salinixanthin, a $\mathrm{C}_{40}$-carotenoid acyl glycoside, serves as a light-harvesting antenna in the retinal-based proton pump xanthorhodopsin of Salinibacter ruber. In the crystallographic structure of this protein, the conjugated chain of salinixanthin is located at the protein-lipid boundary and interacts with residues of helices $\mathrm{E}$ and $\mathrm{F}$. Its ring, with a 4-keto group, is rotated relative to the plane of the $\pi$-system of the carotenoid polyene chain and immobilized in a binding site near the $\beta$-ionone retinal ring. We show here that the carotenoid can be removed by oxidation with ammonium persulfate, with little effect on the other chromophore, retinal. The characteristic CD bands attributed to bound salinixanthin are now absent. The kinetics of the photocycle is only slightly perturbed, showing a 1.5 -fold decrease in the overall turnover rate. The carotenoid-free protein can be reconstituted with salinixanthin extracted from the cell membrane of $S$. ruber. Reconstitution is accompanied by restoration of the characteristic vibronic structure of the absorption spectrum of the antenna carotenoid, its chirality, and the excited-state energy transfer to the retinal. Minor modification of salinixanthin, by reducing the carbonyl $\mathrm{C}=\mathrm{O}$ double bond in the ring to a $\mathrm{C}-\mathrm{OH}$, suppresses its binding to the protein and eliminates the antenna function. This indicates that the presence of the 4-keto group is critical for carotenoid binding and efficient energy transfer.
\end{abstract}

Keywords Light-harvesting - 4-Keto group - Carotenoid binding $\cdot$ Energy transfer $\cdot$ Salinixanthin

E. S. Imasheva · S. P. Balashov $(\bowtie) \cdot$ J. M. Wang · J. K. Lanyi Department of Physiology and Biophysics, C-335 Med Sci I, University of California, Irvine, CA 92697-4560, USA

e-mail: balashov@uci.edu

\section{Introduction}

Light harvesting by carotenoids and other accessory pigments is an essential feature of the bacteriochlorophyll- or chlorophyll-based multipigment and multiprotein photosynthetic systems (Green and Parson 2003; McDermott et al. 1995). The much simpler retinal-based light energy transducers of the archaea, bacteriorhodopsin (Oesterhelt and Stoeckenius 1973) and archaerhodopsin (Mukohata et al. 1991), lack a light-harvesting antenna (Boichenko et al. 2006), though the latter retinal protein contains the carotenoid bacterioruberin at the periphery, which was suggested to play a structural role in the arrangement of the trimers (Yoshimura and Kouyama 2008) and may provide photoprotection as well. Recently, a retinal-based proton pump, xanthorhodopsin (Balashov et al. 2005), was found in the cell membrane of the extremely halophilic eubacterium Salinibacter ruber (Antón et al. 2002). Unlike its archaeal analogues, it contains an accessory light-harvesting antenna, salinixanthin (Balashov et al. 2005). The latter is the main carotenoid of the cell membrane of this organism (Lutnaes et al. 2002). It binds to the retinal protein with a $1: 1$ stoichiometry. About $40-45 \%$ of the quanta absorbed by salinixanthin is transferred to retinal and utilized for proton transport (Balashov et al. 2005, 2008). This approximately doubles the cross section for light absorption by xanthorhodopsin compared to retinal proteins that lack an antenna. The strong absorption bands of salinixanthin in the blue-green region of the spectrum (main maxima at 456, 486 and $521 \mathrm{~nm}$ ), as those of other carotenoids with a long conjugated chain, are from transition to the bright (well-allowed, $1{ }^{1} \mathrm{~B}_{\mathrm{u}}^{+}$-like) state, $\mathrm{S}_{2}$ (Hudson and Kohler 1972; Polívka and Frank 2010; Polívka and Sundström 2004; Schulten and Karplus 1972). This is a short-lived state, with a lifetime of approximately 
66 fs in xanthorhodopsin (Balashov et al. 2008; Polívka et al. 2009) from which fast excitation energy transfer with a time constant of approximately 165 fs (Polívka et al. 2009) takes place, as was shown by steady-state fluorescence (Balashov et al. 2008) and time-resolved femtosecond absorption (Polívka et al. 2009) studies. The acceptor is the allowed excited state of the retinal chromophore that absorbs at approximately $560 \mathrm{~nm}$ (Balashov et al. 2008; Polívka et al. 2009). In retinal proteins with protonated Schiff bases this is the lowest, $\mathrm{S}_{1}, 1^{1} \mathrm{~B}_{\mathrm{u}}^{+}$-like state (Birge and Zhang 1990). Evidence for strong electronic coupling between the two states was gained both in experiments (Balashov et al. 2008; Polívka et al. 2009) and calculations (Fujimoto and Hayashi 2009) that indicated favorable distance and orientation of the two chromophores.

Salinixanthin in the membrane bilayer or in ethanol exhibits the broad absorption spectrum typical of carotenoid compounds with a keto group at the end of the conjugated chain (Britton and Young 1993). It does not show significant circular dichroism (CD) in the visible range (Lutnaes et al. 2002). In contrast, salinixanthin bound to xanthorhodopsin is characterized by a well-resolved vibronic structure in the absorption spectrum (Balashov et al. 2005) and a set of strong bands in the CD spectrum that indicates asymmetric and restricted conformation of the carotenoid in the protein at a specific binding site in which it closely interacts with the retinal (Balashov et al. 2006). Removal of the retinal with hydroxylamine causes broadening of the vibronic structure of the carotenoid and results in a relatively featureless spectrum virtually identical to that of the bulk unbound carotenoid in the membrane. This is accompanied by loss of the CD bands in the visible range (Balashov et al. 2006). Reduction of the Schiff base with sodium borohydride shifts the retinal chromophore band from approximately 560 to $360 \mathrm{~nm}$, but retinal remains attached to the protein and occupies its binding site (Balashov et al. 2008). Under these conditions the carotenoid antenna maintains its well-resolved absorption spectrum (Balashov et al. 2008). Experiments with retinal analogues and retinol showed that carotenoid conformation is restricted sterically by the retinal in the binding site and that the retinal Schiff base linkage is not necessary for defining the carotenoid conformation as long as retinal occupies its binding site (Imasheva et al. 2008; Smolensky and Sheves 2009).

Detailed information about the binding site of the antenna was obtained from the X-ray crystal structure of xanthorhodopsin (Luecke et al. 2008). The carotenoid is buried at the protein-lipid boundary at the outer surface of helix $\mathrm{F}$, at a $54^{\circ}$ angle to the membrane normal. Its keto ring is immobilized by residues at the extracellular ends of helices $\mathrm{E}$ and $\mathrm{F}$ and by the $\beta$-ionone ring of the retinal and rotated from the plane of its polyene chain and, therefore, from the plane of the extended $\pi$-system. This explains the well-resolved vibronic bands of the carotenoid, the lack of a red shift of the bands upon binding and the CD bands in the visible region (Balashov et al. 2006). The ring moieties of the carotenoid and the retinal are within $4 \AA$ of one another. The distance between the centers of salinixanthin and retinal is $11.7 \AA$.

The retinal can be easily removed upon cleavage of the Schiff base with hydroxylamine, and the initial pigment can be reconstituted upon addition of retinal to xanthoopsin (Balashov et al. 2005, 2006; Imasheva et al. 2008). The questions arise whether similar manipulations can be done with the carotenoid moiety, whether the protein is stable without the carotenoid and how the carotenoid modifies the properties of the retinal protein. Reconstitution of light-harvesting complexes from photosynthetic bacteria with carotenoids has provided useful information on their function (Davis et al. 1995; Grabowski et al. 2001; Toropygina et al. 2003) and the factors involved in carotenoid binding (Punginelli et al. 2009; Roszak et al. 2004). Recently, we reconstituted salinixanthin into a protein homologous to xanthorhodopsin, gloeobacter rhodopsin, expressed in Escherichia coli (Imasheva et al. 2009), and showed that substitution of a tryptophan residue in the vicinity of the retinal $\beta$-ionone ring (as in bacteriorhodopsin) with a small glycine (as in xanthorhodopsin and gloeobacter rhodopsin) is a prerequisite for binding of salinixanthin, as predicted from the crystal structure of xanthorhodopsin (Luecke et al. 2008). The latter revealed that the 4-keto ring is in the space that in bacteriorhodopsin is occupied by the bulky Trp138 but that in xanthorhodopsin it is replaced by a Gly.

A number of retinal proteins homologous to xanthorhodopsin and proteorhodopsin from flavobacteria, actinibacteria, proteobacteria and other species bear this feature, which implies that these proteins, pumps and sensors might contain a light-harvesting carotenoid antenna also (Imasheva et al. 2009). This stimulated us to extend our studies of the carotenoid in xanthorhodopsin and its interaction with the retinal protein. As we describe below, we found conditions where incubation of the cell membrane fraction containing xanthorhodopsin with ammonium persulfate removes the salinixanthin antenna but leaves most of the retinal protein intact and stable. This provides an opportunity for future studies of the retinal component of the xanthorhodopsin photocycle in the absence of a contribution from the carotenoid changes normally present in the native pigment (Balashov et al. 2005). By adding salinixanthin, extracted from $S$. ruber, to xanthorhodopsin treated with persulfate, washed and solubilized in n-dodecyl- $\beta$-D-maltopyranoside (DDM), we were able to reconstitute the antenna and energy transfer from it to retinal. We found that reduction of the $\mathrm{C}=\mathrm{O}$ double bond of the 4-keto group in the 
carotenoid ring to a single $\mathrm{C}-\mathrm{OH}$ bond (converting salinixanthin into salinixanthol [Lutnaes et al. 2002]) prevents binding of the carotenoid and energy transfer from it to retinal, indicating that proper geometry of the ring and its keto group is critical to binding.

\section{Materials and Methods}

Culture of $S$. ruber Strain and Isolation of a Fraction of the Cell Membranes Containing Xanthorhodopsin

M31 was grown as described originally by Antón et al. (2002) with some modifications to optimize the yield of xanthorhodopsin (Imasheva et al. 2006).

Isolation was performed as described initially by Balashov et al. (2005) and revised recently (Imasheva et al. 2008). In this latter method the membrane fraction enriched in xanthorhodopsin was separated from the fraction containing salinixanthin not bound to the retinal protein without using a detergent.

\section{Solubilization of Xanthorhodopsin}

Solubilization was done by incubating membrane fractions in a $0.15 \%$ solution of DDM, $50 \mathrm{mM}$ MES (pH 5.7), 100 $\mathrm{mM} \mathrm{NaCl}$ for at least $4 \mathrm{~h}$. Solubilized protein was collected as supernatant after 30-min centrifugation on a bench-top centrifuge (EBA 12R; Hettich, Tuttlingen, Germany) at 14,000 rpm (20,160 RCF).

\section{Extraction of Salinixanthin and Reconstitution}

The carotenoid was extracted from dried membranes with an acetone/methanol (7:3) mixture and separated from proteins and lipids, which were precipitated with cold acetone as described (Lutnaes et al. 2002) and removed by centrifugation. After two to three cycles of such acetone treatment, the solvent was evaporated on a rotary vacuum pump and the carotenoid was dissolved in ethanol and stored at $-60^{\circ} \mathrm{C}$. For reconstitution experiments the salinixanthin was dispersed in $0.15 \% \mathrm{DDM}, 100 \mathrm{mM} \mathrm{NaCl}$.

\section{Reduction of the Salinixanthin 4-Keto Group} with Sodium Borohydride

Addition of sodium borohydride to an ethanol solution of salinixanthin $(5 \mathrm{mg}$ to $1.5 \mathrm{ml}$ ) causes reduction of its 4-keto group to a 4-hydroxy group (salinixanthol) (Lutnaes et al. 2002). The reaction was monitored spectroscopically, following the appearance of a well-resolved structure in the absorption spectrum and a shift of the maxima from 458 , 486 and $521 \mathrm{~nm}$ to 447,475 and $506 \mathrm{~nm}$ caused by elimination of the 4-keto group, which is known to cause broadening of the absorption when conjugated to the main chain of a carotenoid (Britton 1995). To insure maximal transformation of salinixanthin to salinixanthol, incubation with borohydride continued until no changes were seen in the spectra, which was about $2 \mathrm{~h}$. The amount of remaining salinixanthin could be estimated from the amplitude of a sharp band at $320 \mathrm{~nm}$ in the second derivative spectrum. The unreacted crystalline borohydride was separated by sedimentation using a bench-top centrifuge (Hettich EBA $12 \mathrm{R})$ at $14,000 \mathrm{rpm}(20,160 \mathrm{RCF})$ for $5 \mathrm{~min}$. Ethanol was evaporated and the carotenoid dissolved in $0.15 \%$ DDM, $100 \mathrm{mM} \mathrm{NaCl}$. To allow traces of borohydride to react with water and exhaust itself, the sample was incubated overnight. Part of the salinixanthol was present in an aggregated form, especially at $\mathrm{pH} 5.7$ and less so at $\mathrm{pH}$ 7.2. Aggregates were separated by centrifugation. These aggregates contributed about $20 \%$ to the absorption spectrum but produced a strong $\mathrm{CD}$ signal, with a maximum around $370 \mathrm{~nm}$. After separation, the amplitude of this band in the CD spectrum was reduced by more than 10 -fold and did not interfere with changes upon reconstitution.

\section{Spectral Measurements}

Absorption spectra were measured on a Shimadzu (Kyoto, Japan) UV 1700 spectrophotometer in quartz cuvettes. CD spectra were obtained on a Jasco (Tokyo, Japan) 720 spectropolarimeter as described (Balashov et al. 2006). Typically, spectra were recorded with 1-nm steps at a scan speed of $50 \mathrm{~nm} / \mathrm{min}$ and a $4-\mathrm{s}$ time constant with a $2-\mathrm{nm}$ bandwidth and averaging of 16 spectra. Fluorescence excitation spectra were obtained on an SLM spectrofluorometer as described earlier (Balashov et al. 2008). The excitation bandwidth was $8 \mathrm{~nm}$. Retinal chromophore emission was measured at $720 \mathrm{~nm}$. The presence of additional fluorescing centers from unidentified contamination was found with an emission peak at $620 \mathrm{~nm}$. In the initial samples and especially after carotenoid bleaching, an excitation band at $450-460 \mathrm{~nm}$ was observed for this emission and, to a much lesser extent, for 720-nm emission. This band was subtracted after appropriate scaling.

\section{Kinetics of Laser Pulse-Induced Absorption Changes}

The kinetics of photocycle reactions was examined using a pulsed laser-based flash photometer (Sure Lite I, excitation at $532 \mathrm{~nm}, 7 \mathrm{~ns}$; Continuum, Santa Clara, CA), with a double monochromator in the measuring beam. The data were fit to a sum of exponential components using the FITEXP program (Dioumaev 1997; Sharonov et al. 1991), kindly provided by Dr. A. K. Dioumaev. 


\section{Results and Discussion}

Oxidation of Salinixanthin in Xanthorhodopsin with Ammonium Persulfate

In search of an agent that could selectively oxidize and remove the carotenoid from its binding site in aqueous suspensions of xanthorhodopsin, we found that ammonium persulfate was effective. It bleached the salinixanthin not bound to xanthorhodopsin and yielded a product absorbing in the near UV region (Fig. 1); it was effective in xanthorhodopsin also (Fig. 2). Figure 2a shows absorption changes in a suspension of cell membranes containing xanthorhodopsin upon incubation with $5 \mathrm{mM}$ ammonium persulfate. The characteristic well-resolved absorption bands of bound salinixanthin at 456, 486 and $521 \mathrm{~nm}$ disappeared, while most of the retinal protein band remained. The difference spectra in Fig. $2 b$ and the
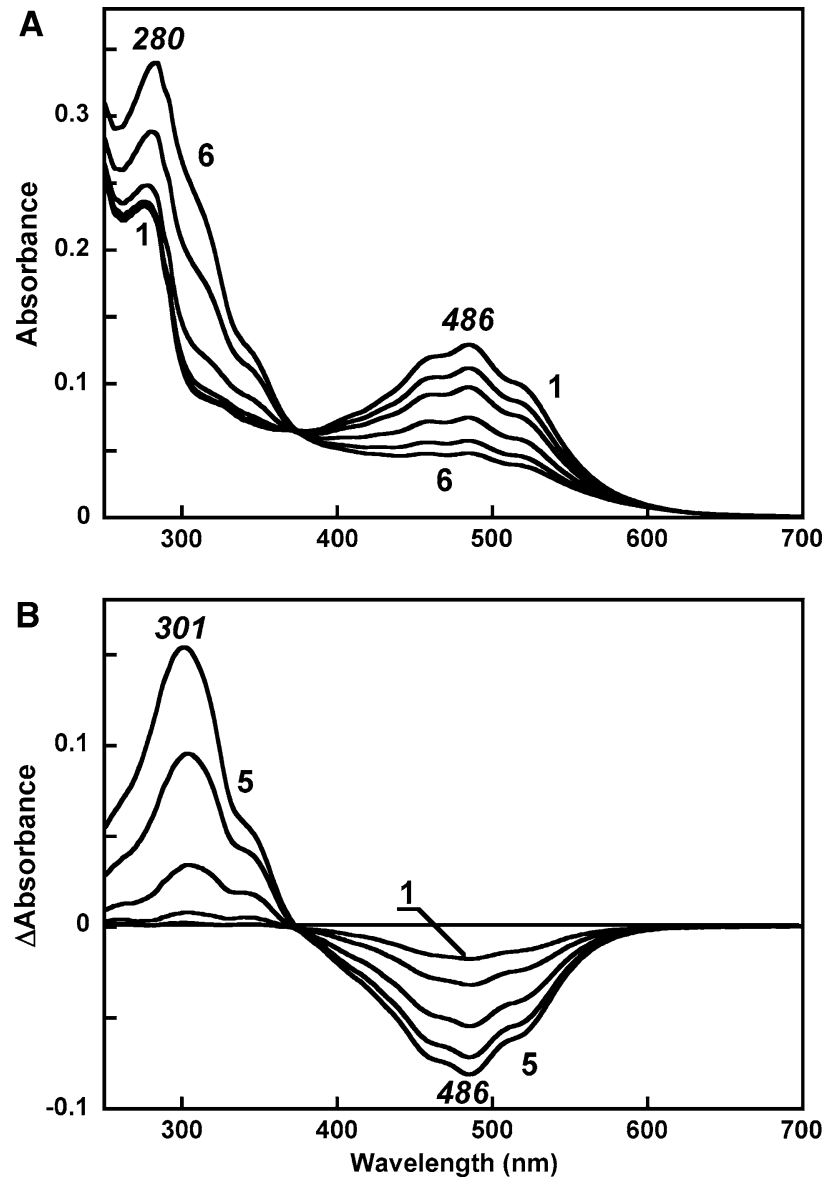

Fig. 1 Reaction of membrane fraction containing salinixanthin not bound to xanthorhodopsin with $5 \mathrm{mM}$ persulfate in the dark, $\mathrm{pH}$ 5.7. a Absorption spectra of suspension measured before (1) and after (2-6) addition of $5 \mathrm{mM}$ persulfate and incubation for 1, 2, 4, 8 and $12 \mathrm{~h}$. b Corresponding difference spectra "spectrum $i$ minus spectrum 1 ," where $i=\operatorname{spectra} 2-6$ in $\mathbf{a}$ kinetic traces in Fig. 2c show that changes in the retinal band (in the 600-nm region) do occur but are relatively small compared to the changes in the carotenoid bands. They indicate that the amplitude of the retinal chromophore band decreases by about $14 \%$ after 10 -h incubation with ammonium persulfate at $\mathrm{pH} 5.7$, whereas about $98 \%$ of carotenoid was bleached. The time constant for the carotenoid bleaching varied in different preparations between $1.5 \mathrm{~h}$ (as in Fig. 2) and $5 \mathrm{~h}$. The amount of remaining carotenoid is easy to estimate from the amplitude of the second derivative of the absorption spectrum (Fig. 2d) because in the second derivative the narrow carotenoid bands dominate and the broader retinal bands become visible only when most of the carotenoid is bleached. The maximum of the retinal chromophore absorption band in the absence of the carotenoid is at 551-553 nm, as determined in the absolute absorption spectrum (Fig. 2a, curve 5). The pigment without carotenoid is stable. As the initial xanthorhodopsin, it can be bleached additionally by hydroxylamine to remove the retinal as well (Fig. 3). The difference spectrum shows a positive band from retinal oxime at $360 \mathrm{~nm}$ and a negative band at $551 \mathrm{~nm}$ (Fig. 3, curve 1) from the retinal chromophore. In contrast, without prior treatment with ammonium persulfate, bleaching of the retinal band by hydroxylamine is accompanied by changes in the carotenoid spectrum (curve 2). The question arises whether removal of the carotenoid or treatment of the membranes with ammonium persulfate affects the position of the retinal band. Absorption changes presented in Fig. $2 \mathrm{a}, \mathrm{b}$ indicates that $10-\mathrm{h}$ incubation with ammonium persulfate results only in a small (less than $5 \mathrm{~nm}$ ) blue shift of the retinal band.

Properties of Xanthorhodopsin with the Carotenoid Bleached: CD Spectra and Kinetics of the Photocycle

Figure 4 shows that the sharp intense bands in the CD spectrum of native xanthorhodopsin (spectrum 1) are eliminated after bleaching of salinixanthin with persulfate, as expected from their assignment to the antenna component (Balashov et al. 2006). The broader and less intense band in the visible range seen in the bleached sample (curve 2) is from the retinal chromophore, due to its asymmetric conformation (twists in the polyene chain) and/or an asymmetric environment in its binding site. The broad single band of the retinal chromophore, with a maximum at approximately $550 \mathrm{~nm}$, has a shape different from that of bacteriorhodopsin, where a bilobe was observed, associated with the trimeric unit of the purple membrane (Heyn et al. 1975; Wu and El-Sayed 1991). Xanthorhodopsin with bleached salinixanthin, although in the membrane, exhibits a CD 

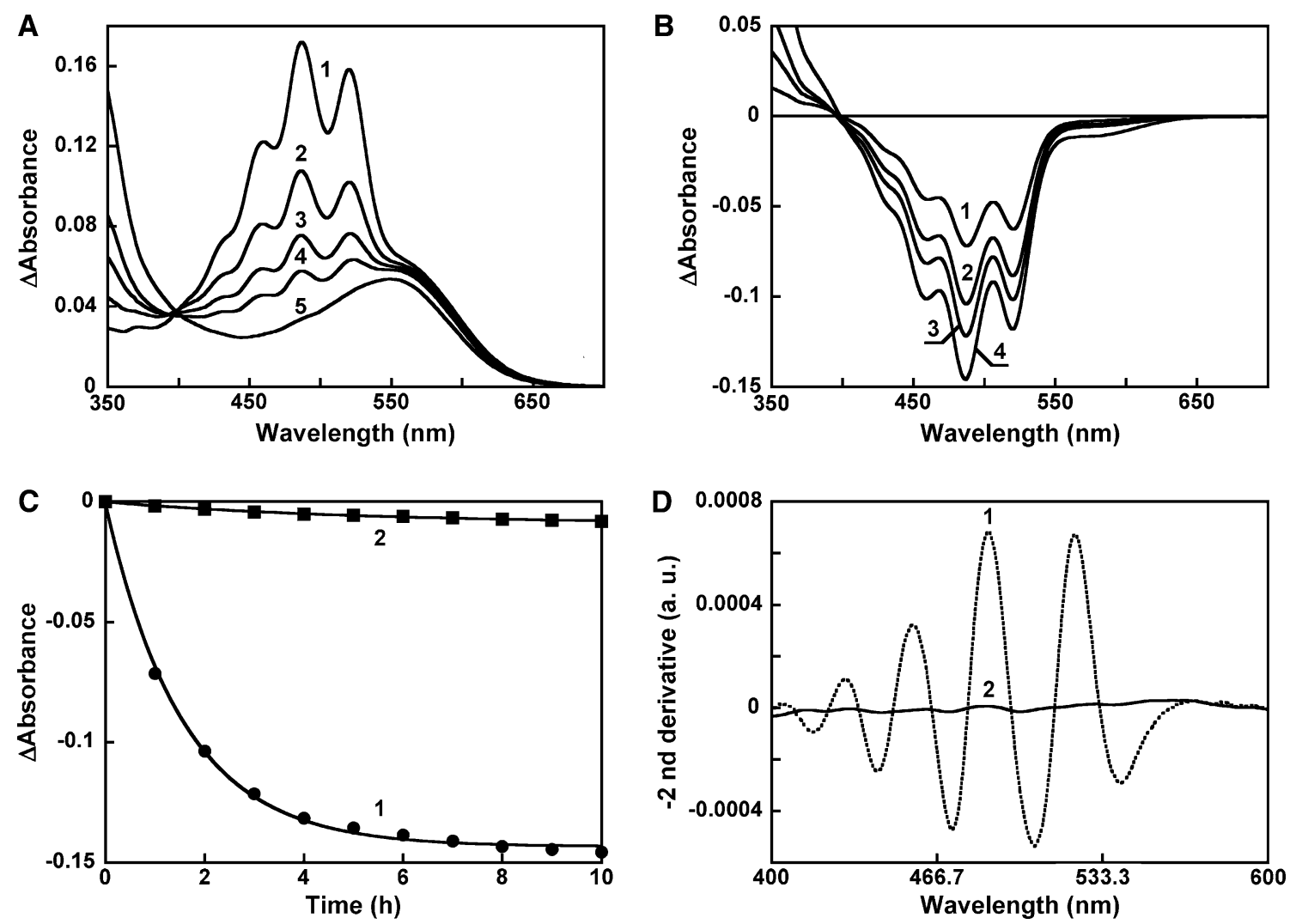

Fig. 2 Bleaching (oxidation) of salinixanthin in suspension of $S$. ruber cell membranes containing xanthorhodopsin upon incubation with $5 \mathrm{mM}$ ammonium persulfate in the dark at $\mathrm{pH}$ 5.7. a 1-5, absorption spectra measured before and after incubation for 1, 2, 3 and $10 \mathrm{~h}$, respectively. b $1-4$, absorption changes caused by

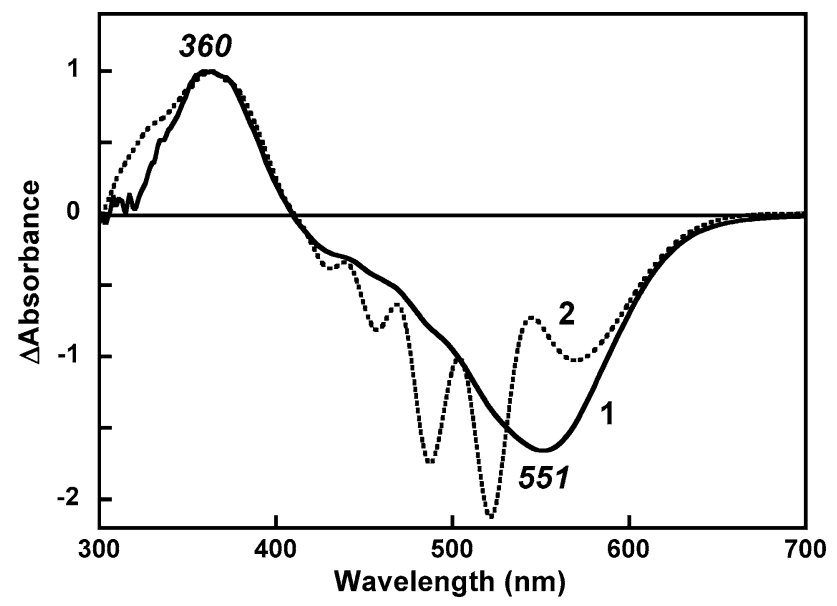

Fig. 3 Difference absorption spectra obtained upon illumination with 550-650 nm light of membranes containing xanthorhodopsin in the presence of $0.2 \mathrm{M}$ hydroxylamine, $\mathrm{pH}$ 7.2: spectrum 1, membranes after bleaching of carotenoids with ammonium persulfate; spectrum 2, membranes without ammonium persulfate treatment. Spectra were normalized at $360 \mathrm{~nm}$

spectrum like the spectrum of detergent-solubilized bacteriorhodopsin monomers (Becher and Ebrey 1976; Heyn et al. 1975).

incubation with ammonium persulfate for $1,2,3$ and $10 \mathrm{~h}$, respectively. c Kinetics of absorption changes at 1, 486 and 2, 600 $\mathrm{nm}$. d Second derivatives of the absorption spectra before (1) and after (2) incubation with ammonium persulfate for $10 \mathrm{~h}$

In order to test whether incubation of xanthorhodopsin with persulfate and oxidation of the carotenoid affect the photocycle reactions of the retinal protein, we examined the kinetics of transient absorption changes at three characteristic wavelengths $(410,570$ and $620 \mathrm{~nm})$ after flash photoexcitation, both in the untreated membranes at $\mathrm{pH} 8.6$ and after bleaching of salinixanthin. The results are presented in Fig. 5 and Table 1. The kinetics of the photocycle in xanthorhodopsin (Balashov et al. 2005) and the preparation treated with ammonium persulfate can be satisfactorily described with six kinetic components. They show no major effects from carotenoid bleaching, but some changes do occur. The decay of the primary photoproduct $\mathrm{K}$ followed at $620 \mathrm{~nm} \tau_{1}$ and formation of the $\mathrm{M}$ intermediate monitored at $410 \mathrm{~nm}$ and characterized mostly by $\left(\tau_{2}\right)$ were slightly slower. The decay of the $\mathrm{M}$ intermediate $\left(\tau_{3}\right)$ accelerated upon carotenoid removal, with the time constant decreasing from 230 to $144 \mu \mathrm{s}$. The largest change was observed at the end of the photocycle, which resulted in a decrease in the rate of recovery of the initial state $\left(\tau_{6}\right)$ monitored at $570 \mathrm{~nm}$, from 209 to $343 \mathrm{~ms}$. The carotenoid undergoes perturbations during the photocycle as follows from two different kinds of spectral changes of carotenoid 


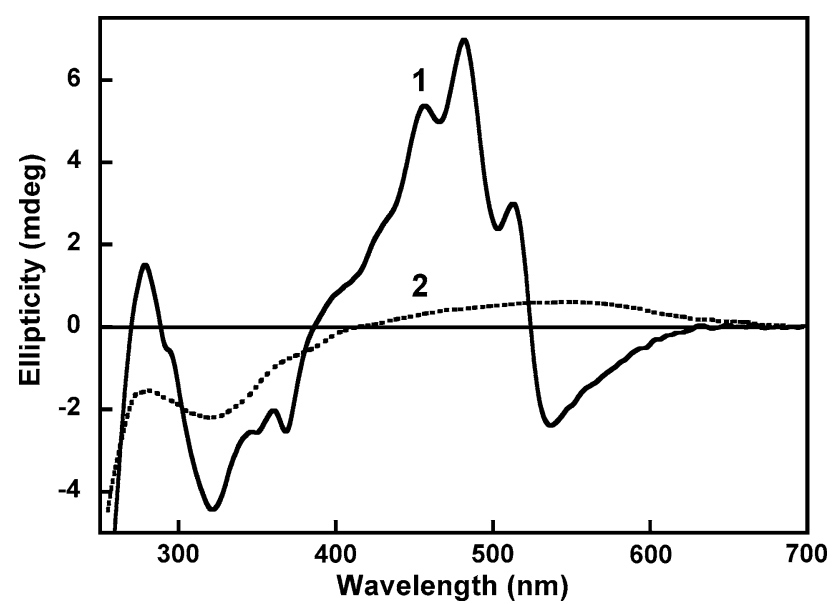

Fig. 4 Effect of carotenoid bleaching on the $\mathrm{CD}$ spectrum of xanthorhodopsin. Spectrum 1, initial membranes containing xanthorhodopsin, $\mathrm{pH}$ 5.7; spectrum 2, after bleaching of carotenoids with ammonium persulfate

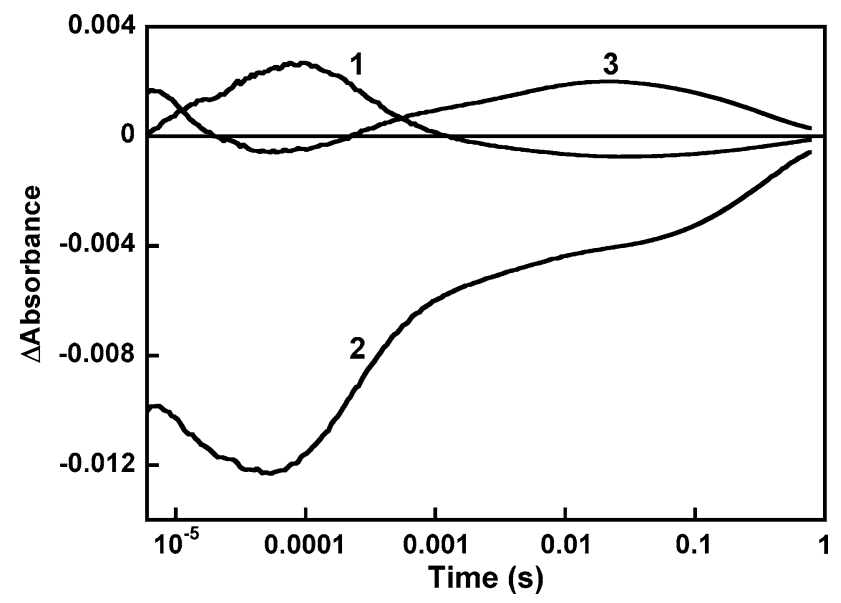

Fig. 5 Kinetics of laser flash-induced absorption changes in the sample after bleaching of salinixanthin with $5 \mathrm{mM}$ persulfate, at $\mathrm{pH}$ 8.6 at three wavelengths: 1,$410 ; 2,570$; and $3,620 \mathrm{~nm}$

Table 1 Kinetic components

\begin{tabular}{lllllll}
\hline & $\begin{array}{l}\tau_{1} \\
(\mu \mathrm{s})\end{array}$ & $\begin{array}{l}\tau_{2} \\
(\mu \mathrm{s})\end{array}$ & $\begin{array}{l}\tau_{3} \\
(\mu \mathrm{s})\end{array}$ & $\begin{array}{l}\tau_{4} \\
(\mu \mathrm{s})\end{array}$ & $\begin{array}{l}\tau_{5} \\
(\mathrm{~ms})\end{array}$ & $\begin{array}{l}\tau_{6} \\
(\mathrm{~ms})\end{array}$ \\
\hline $\begin{array}{l}\text { Initial XR in } \\
\text { membrane }\end{array}$ & 7.3 & 36 & 230 & 681 & 5.3 & 209 \\
$\begin{array}{l}\text { Treated with APS } \\
\text { reated }\end{array}$ & 8.2 & 45 & 144 & 508 & 5.0 & 343 \\
\hline
\end{tabular}

bands observed upon formation of the primary photoproduct $\mathrm{K}$ and at the end of the photocycle (Balashov et al. 2005). This would be expected from the close approach of the carotenoid keto ring to the retinal ionone ring and the location of the conjugated chain across helix $\mathrm{F}$, which makes it susceptible to chromophore isomerization, movement of helix $\mathrm{F}$ and local electric fields during proton transfer. On the other hand, removal of the carotenoid and some loosening of the retinal binding site (at least in the ring area) might decrease the rate of reisomerization of the retinal chromophore and restoration of the initial state.

Reconstitution of Salinixanthin into Xanthorhodopsin Treated with Ammonium Persulfate

Addition of salinixanthin in ethanol solution to a suspension of cell membranes containing xanthorhodopsin bleached with ammonium persulfate does not cause significant changes in the carotenoid spectrum, indicating that no reconstitution takes place. The reason could be that the carotenoid is not sufficiently soluble for partitioning into the membranes or that the binding site is occupied by fragments from the oxidized carotenoid. Specific binding of the carotenoid does occur, however, after solubilization of the membranes in DDM. This is shown in Fig. 6a. Solubilization of xanthorhodopsin treated with ammonium persulfate shifts the maximum of the retinal band to 546 $\mathrm{nm}$ at $\mathrm{pH} 5.7$ (spectrum 1). Salinixanthin dissolved in $0.15 \%$ DDM shows a spectrum with a poorly resolved vibronic structure (spectrum 2), as in an organic solvent. However upon addition to xanthorhodopsin, the characteristic fine structure of the spectrum with maxima at 521, 486 and $456 \mathrm{~nm}$ reappeared, which indicates specific binding. Subsequent additions of aliquots of salinixanthin caused a further increase in the fraction of bound salinixanthin until all of the protein was reconstituted (Fig. 6b). The rate of reconstitution (which is likely limited by the rate of fusion of micelles containing xanthorhodopsin and salinixanthin) depends on salt concentration. In $100 \mathrm{mM}$ salt, reconstitution occurs within a few minutes. In the absence of salt, it occurs within $1 \mathrm{~h}$, and one can follow the binding of the carotenoid by measuring the difference spectra after addition of salinixanthin to xanthorhodopsin, as shown in Fig. 6c. The spectral changes upon carotenoid binding are characterized by an increase in absorbance in the main carotenoid bands at 518, 485 and $454 \mathrm{~nm}$ and a decrease at $543 \mathrm{~nm}$ from sharpening of the vibrational bands. These changes are accompanied by a decrease of the 326 and $381 \mathrm{~nm}$ bands, apparently from a conformational change of the carotenoid chain in the bound state, and an increase of absorbance at $296 \mathrm{~nm}$ from perturbation of aromatic residues in the carotenoid and retinal binding site. Reconstitution of the carotenoid under these conditions (no salt) takes place with a time constant of about $20 \mathrm{~min}$, as indicated by the kinetics of absorption changes in the main carotenoid band (see Fig. 6c, inset).

The fraction of the reconstituted pigment was estimated from the amplitude of the second derivative spectrum from bound carotenoid as described (Imasheva et al. 2009). It suggests that approximately $85 \%$ of the pigment was 

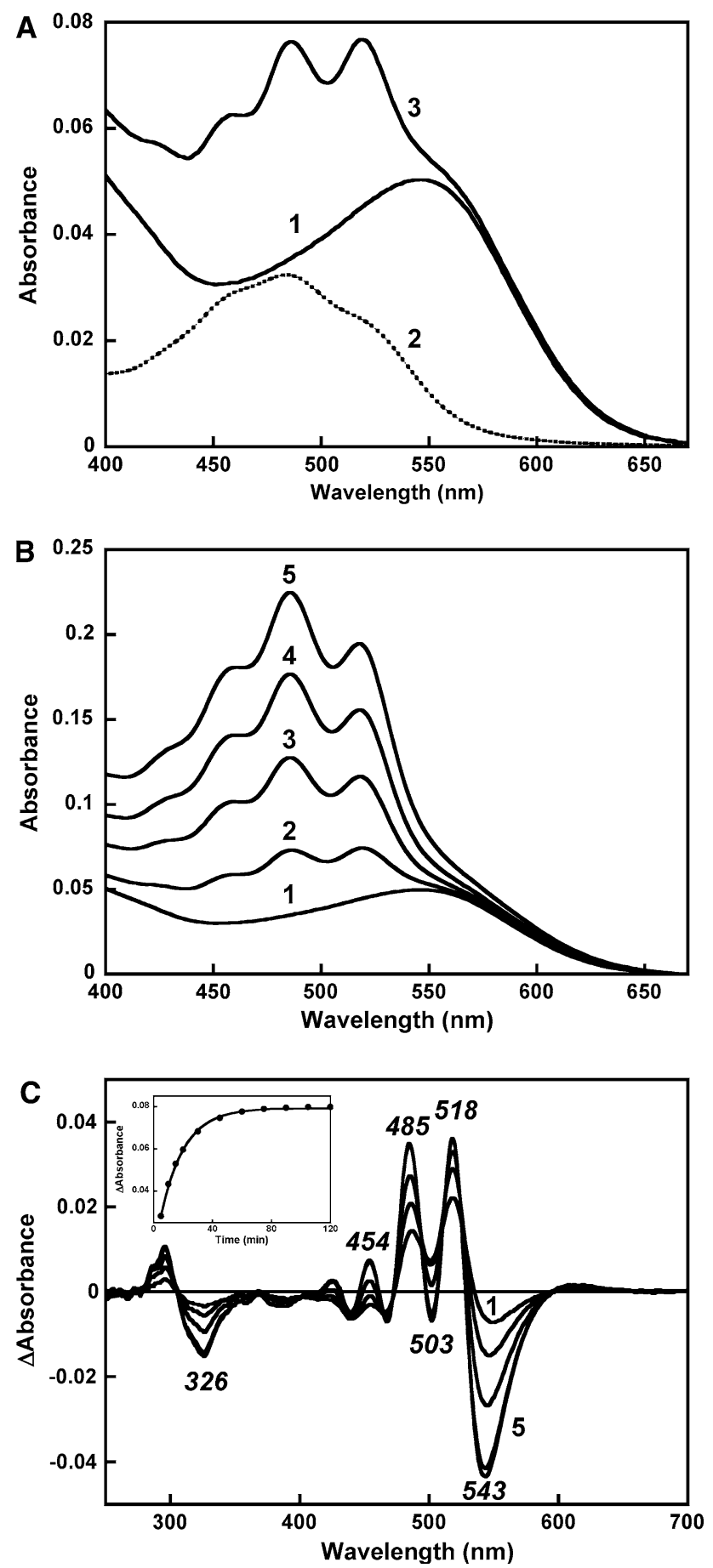

Fig. 6 Absorption changes accompanying reconstitution of salinixanthin into xanthorhodopsin. a Absorption spectra of $1,2.5 \mu \mathrm{M}$ xanthorhodopsin bleached with ammonium persulfate and solubilized in $0.15 \%$ DDM, $50 \mathrm{mM}$ MES ( $\mathrm{pH} 5.7$ ), $100 \mathrm{mM} \mathrm{NaCl} ; 2,0.7 \mu \mathrm{M}$ salinixanthin in $0.15 \%$ DDM, $50 \mathrm{mM}$ MES (pH 5.7), $100 \mathrm{mM} \mathrm{NaCl}$; and 3 , after mixing samples 1 and 2. b $1-5$, absorption spectra obtained upon consecutive additions of $0.7 \mu \mathrm{M}$ of salinixanthin to 2.5 $\mu \mathrm{M}$ xanthorhodopsin. Conditions as in a. c 1-5, absorption changes observed after addition of salinixanthin to xanthorhodopsin bleached with persulfate, measured after 5, 10, 20, 60 and $90 \mathrm{~min}$, respectively, in $0.15 \%$ DDM, $50 \mathrm{mM}$ MES (pH 5.7); no salt added

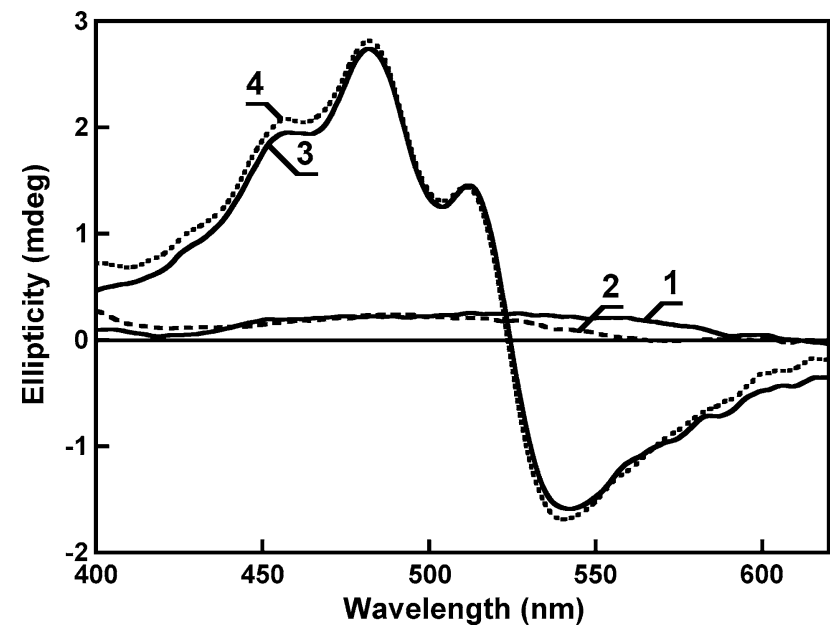

Fig. 7 Restoration of the CD spectrum after reconstitution of xanthorhodopsin with salinixanthin. Spectrum 1 , xanthorhodopsin after bleaching of the carotenoid component with persulfate and solubilization in $0.15 \%$ DDM; spectrum 2, salinixanthin in $0.15 \%$ DDM; spectrum 3, after reconstitution (mixing samples 1 and 2); spectrum 4, initial (unbleached) xanthorhodopsin solubilized in $0.15 \%$ DDM, $50 \mathrm{mM}$ MES ( $\mathrm{pH} 5.7$ ), $100 \mathrm{mM} \mathrm{NaCl}$, scaled to spectrum 3

reconstituted upon addition of carotenoid in a 1.5:1 carotenoid/retinal ratio.

The CD spectrum of reconstituted pigment showed all of the features of the native xanthorhodopsin (Fig. 7, spectrum 3). This provides additional evidence that carotenoid entered its binding site and assumed the native conformation. Most important is that this correlates with restoration of the antenna function. As shown in Fig. 8d, the excitation spectrum for the retinal chromophore emission of the sample after carotenoid bleaching and solubilization is a broad band with a maximum at $550 \mathrm{~nm}$. After reconstitution with salinixanthin, sharp bands appear at 522, 488 and $456 \mathrm{~nm}$, indicating energy transfer from bound carotenoid to the retinal chromophore. In addition, some increase in the red region of the retinal band is observed, which might indicate that carotenoid binding affects the fraction of the pigment with protonated counterion.

\section{Reconstitution with Salinixanthol}

The ability to remove and reconstitute salinixanthin into xanthorhodopsin opens the possibility of performing experiments with modified carotenoids, which could lead to understanding of the structural requirements for their binding. An intriguing question is whether the keto group is essential for binding and excited-state energy transfer. We addressed this question by converting salinixanthin into salinixanthol by reducing the $\mathrm{C}=\mathrm{O}$ carbonyl to a $\mathrm{C}-\mathrm{OH}$ group with sodium borohydride. The difficulty of the 

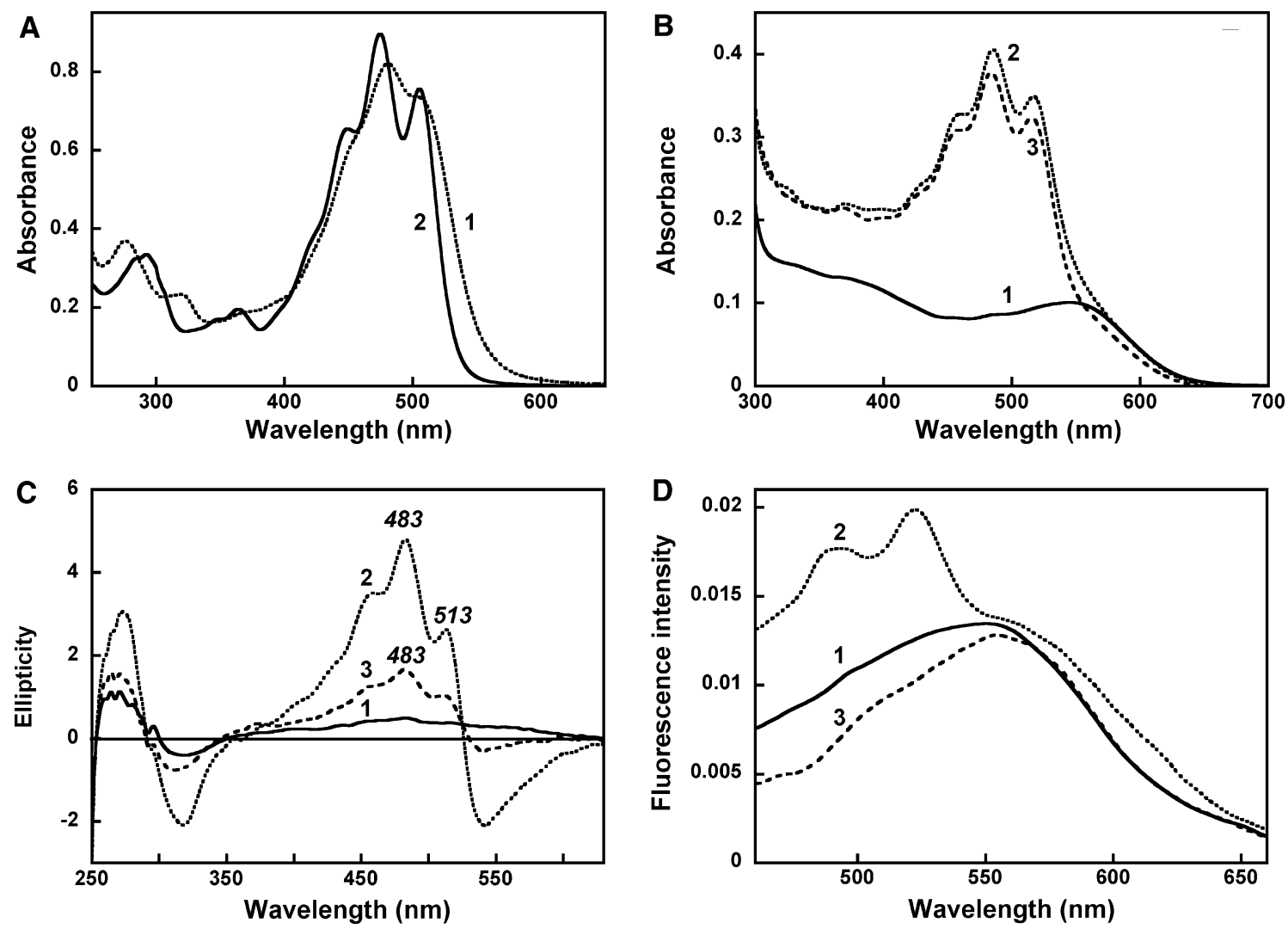

Fig. 8 Comparison of spectral features upon reconstitution of xanthorhodopsin with salinixanthin and salinixanthol. a Absorption spectra in ethanol of 1 , salinixanthin and 2, salinixanthol. b Absorption spectra in $0.15 \%$ DDM (pH 5.7) of 1 , xanthorhodopsin after treatment with persulfate and solubilization; 2, reconstituted with salinixanthin and 3, mixed with salinixanthol. c CD spectra of

experiments with salinixanthol is that, unlike salinixanthin, it has an absorption spectrum with well-resolved vibronic structure, as was pointed out by Lutnaes et al. (2002) and as shown in Fig. 8a. If specific binding occurs, it might not be accompanied by any significant changes in vibrational structure of the carotenoid, as observed when pigment is reconstituted with salinixanthin (Fig. 6a). Indeed, addition of salinixanthol to xanthorhodopsin bleached with persulfate did not cause any large changes in resolution of vibronic bands, but that did not rule out binding. The spectrum of the mixture of salinixanthol with xanthorhodopsin with removed native carotenoid in fact is very similar to the spectrum of the protein reconstituted with salinixanthin (Fig. 8b). The assay for binding could be from changes in the $\mathrm{CD}$ spectrum. Figure $8 \mathrm{c}$ shows that reconstitution of xanthorhodopsin with salinixanthol results only in partial restoration of the $\mathrm{CD}$ spectrum, 27\% in amplitude compared to when pure salinixanthin was added. It should be noted that the borohydride reduction of salinixanthin to salinixanthol left approximately 15\% unreacted pigment samples 1-3 described in b. d Excitation spectra for emission at 720 $\mathrm{nm}$ for samples 1-3 described in $\mathbf{b}$. Absorption of the samples was $0.3,0.24$ and 0.23 at the maximum, respectively. Contribution from an unknown fluorescing center (maximum emission at $610 \mathrm{~nm}$, maximum in the excitation spectrum at $456 \mathrm{~nm}$ ) was subtracted. Spectra are scaled to their maxima

and that about half of the CD amplitude comes from the remaining salinixanthin. Thus, salinixanthol is at least eight times less efficient in binding than salinixanthin. Independent evidence for poor binding of salinixanthol was obtained from excitation spectra for retinal fluorescence emission. When reconstituted with salinixanthin, additional bands at 522 and $488 \mathrm{~nm}$ appeared in the excitation spectrum. No such bands were seen when reconstitution was made with salinixanthol but, rather, some decrease in the amplitude of the spectrum at wavelengths where salinixanthol absorbs was observed, apparently from a screening effect of added carotenoid (Fig. 8d). This indicates that no (or very little) energy transfer occurs from salinixanthol to retinal, suggesting that either the $\mathrm{C}=\mathrm{O}$ group is required for efficient energy transfer or the occupancy of salinixanthol is very low in the binding site, even after long incubation. It might occur for steric reasons, e.g., when the $\mathrm{C}-\mathrm{OH}$ group of the ring does not fit the binding site or the ring has a different conformation. Another possibility is that the $\mathrm{C}=\mathrm{O}$ group is engaged in interactions in the binding site which 
strongly facilitate binding of the carotenoid, though the crystal structure did not reveal any hydrogen bonding (Luecke et al. 2008).

In conclusion, the results indicate that the carotenoid antenna can be removed from xanthorhodopsin and then reconstituted. Binding and energy transfer critically depend on the presence of a $\mathrm{C}=\mathrm{O}$ carbonyl group in the carotenoid ring.

Acknowledgments This work was supported in part by grants from the National Institutes of Health (GM29498) and the Department of Energy (DEFG03-86ER13525) to J. K. L. and the U.S. Army Research Office (W911NF-09-1-0243) to S. P. B.

Open Access This article is distributed under the terms of the Creative Commons Attribution Noncommercial License which permits any noncommercial use, distribution, and reproduction in any medium, provided the original author(s) and source are credited.

\section{References}

Antón J, Oren A, Benlloch S et al (2002) Salinibacter ruber gen. nov., sp. nov., a novel, extremely halophilic member of the Bacteria from saltern crystallizer ponds. Int $\mathrm{J}$ Syst Evol Micrbiol $52: 485-491$

Balashov SP, Imasheva ES, Boichenko VA et al (2005) Xanthorhodopsin: a proton pump with a light-harvesting carotenoid antenna. Science 309:2061-2064

Balashov SP, Imasheva ES, Lanyi JK (2006) Induced chirality of the light-harvesting carotenoid salinixanthin and its interaction with the retinal of xanthorhodopsin. Biochemistry 45:10998-11004

Balashov SP, Imasheva ES, Wang JM et al (2008) Excitation energytransfer and the relative orientation of retinal and carotenoid in xanthorhodopsin. Biophys J 95:2402-2414

Becher B, Ebrey TG (1976) Evidence for chromophore-chromophore (exciton) interaction in the purple membrane of Halobacterium halobium. Biochem Biophys Res Commun 69:1-6

Birge RR, Zhang C-F (1990) Two-proton double resonance spectroscopy of bacteriorhodopsin. Assignment of the electronic and dipolar properties of the low-lying ${ }^{1} \mathrm{~A}_{\mathrm{g}}{ }^{*}$-like and ${ }^{1} \mathrm{~B}_{\mathrm{u}}{ }^{*+}$-like $\pi$, $\pi^{*}$ states. J Chem Phys 92:7178-7195

Boichenko VA, Wang JM, Antón J et al (2006) Functions of carotenoids in xanthorhodopsin and archaerhodopsin, from action spectra of photoinhibition of cell respiration. Biochim Biophys Acta 1757:1649-1656

Britton G (1995) UV/visible spectroscopy. In: Britton G, LiaaenJensen S, Pfander H (eds) Carotenoids, vol 1B. Spectroscopy. Birkhäuser Verlag, Basel, Boston, Berlin, pp 13-62

Britton G, Young AJ (1993) Methods for isolation and analysis of carotenoids. In: Young AJ, Britton G (eds) Carotenoids in photosynthesis. Chapman \& Hall, London, pp 409-457

Davis CM, Bustamante PL, Loach PA (1995) Reconstitution of bacterial core light-harvesting complexes of Rhodobacter sphaeroides and Rhodospirillum rubrum with isolated $\alpha$ - and $\beta$-polypeptides, bacteriochlorophyll $a$ and carotenoid. J Biol Chem 270:5793-5804

Dioumaev AK (1997) Evaluation of intrinsic chemical kinetics and transient product spectra from time-resolved spectroscopic data. Biophys Chem 67:1-25
Fujimoto KJ, Hayashi S (2009) Electronic coulombic coupling of excitation-energy transfer in xanthorhodopsin. J Am Chem Soc 131:14152-14153

Grabowski B, Cunningham FX, Gantt E (2001) Chlorophyll and carotenoid binding in a simple red algal light-harvesting complex crosses phylogenetic lines. Proc Natl Acad Sci USA 98:2911-2916

Green BR, Parson WW (2003) Light-harvesting antennas in photosynthesis. In: Govindjee (ed) Advances in photosynthesis and respiration. Kluwer Academic, Dordrecht

Heyn MP, Bauer P-J, Dencher NA (1975) A natural CD label to probe the structure of the purple membrane from Halobacterium halobium by means of exciton coupling effects. Biochem Biophys Res Commun 67:897-903

Hudson BS, Kohler BE (1972) A low-lying weak transition in the polyene $\alpha \omega$-diphenyloctatetraene. Chem Phys Lett 14:299-304

Imasheva ES, Balashov SP, Wang JM et al (2006) pH-dependent transitions in xanthorhodopsin. Photochem Photobiol 82:1406-1413

Imasheva ES, Balashov SP, Wang JM et al (2008) Chromophore interaction in xanthorhodopsin-retinal dependence of salinixanthin binding. Photochem Photobiol 84:977-984

Imasheva ES, Balashov SP, Choi AR et al (2009) Reconstitution of Gloeobacter violaceus rhodopsin with a light-harvesting carotenoid antenna. Biochemistry 48:10948-10955

Luecke H, Schobert B, Stagno J et al (2008) Crystallographic structure of xanthorhodopsin, the light-driven proton pump with a dual chromophore. Proc Natl Acad Sci USA 105:16561-16565

Lutnaes BF, Oren A, Liaaen-Jensen S (2002) New $\mathrm{C}_{40}$-carotenoid acyl glycoside as principal carotenoid in Salinibacter ruber, an extremely halophilic eubacterium. J Nat Prod 65:1340-1343

McDermott G, Prince SM, Freer AA et al (1995) Crystal structure of an integral membrane light-harvesting complex from photosynthetic bacteria. Nature 374:517-521

Mukohata Y, Ihara K, Uegaki K et al (1991) Australian Halobacteria and their retinal-protein ion pumps. Photochem Photobiol 54:1039-1045

Oesterhelt D, Stoeckenius W (1973) Functions of a new photoreceptor membrane. Proc Natl Acad Sci USA 70:2853-2857

Polívka T, Frank HA (2010) Molecular factors controlling photosynthetic light harvesting by carotenoids. Acc Chem Res 43:1125-1134

Polívka T, Sundström V (2004) Ultrafast dynamics of carotenoid excited states-from solution to natural and artificial systems. Chem Rev 104:2021-2071

Polívka T, Balashov SP, Chábera P et al (2009) Femtosecond carotenoid to retinal energy transfer in xanthorhodopsin. Biophys J 96:2268-2277

Punginelli C, Wilson A, Routaboul JM et al (2009) Influence of zeaxanthin and echinenone binding on the activity of the orange carotenoid protein. Biochim Biophys Acta 1787:280-288

Roszak AW, McKendrick K, Gardiner AT et al (2004) Protein regulation of carotenoid binding: gatekeeper and locking amino acid residues in reaction centers of Rhodobacter sphaeroides. Structure 12:765-773

Schulten K, Karplus M (1972) On the origin of a low-lying forbidden transition in polyenes and related molecules. Chem Phys Lett 14:305-309

Sharonov AY, Tkachenko NV, Savransky VV et al (1991) Timeresolved ultraviolet-absorption changes in the photocycle of bacteriorhodopsin. Photochem Photobiol 54:889-895

Smolensky E, Sheves M (2009) Retinal-salinixanthin interactions in xanthorodopsin: a circular dichroism (CD) spectroscopy study with artificial pigments. Biochemistry 48:8179-8188 
Toropygina OA, Makhneva ZK, Moskalenko AA (2003) Reconstitution of carotenoids into the light-harvesting complex B800-850 of Chromatium minutissimum. Biochemistry (Mosc) 68:901-911

Wu SG, El-Sayed MA (1991) CD spectrum of bacteriorhodopsinbest evidence against exciton model. Biophys J 60:190-197
Yoshimura K, Kouyama T (2008) Structural role of bacterioruberin in the trimeric structure of archaerhodopsin-2. J Mol Biol 375: $1267-1281$ 